\title{
ARTICLE
}

Cite this: DOI: $10.1039 /$ xoxxooooox

\section{Functionalized protein nanoemulsions by incorporation of chemically modified BSA}

Received ooth January 2012, Accepted ooth January 2012

DOI: $10.1039 / \times 0 \times x 00000 x$

www.rsc.org/

\author{
Ana Loureiro, ${ }^{a, b}$ Ana S. Abreu, ${ }^{a, \dagger}$ Marisa Passos Sárria,,${ }^{a, b}$ Mafalda C. O. \\ Figueiredo, ${ }^{c}$ Lígia M Saraiva, ${ }^{c}$ Gonçalo J. L. Bernardes, ${ }^{d, e}$ Andreia C. Gomes ${ }^{b}$ \\ and Artur Cavaco-Paulo ${ }^{a *}$
}

The incorporation of bioactive compounds in stealth nanoparticles or nanoemulsions enhances their half-life in systemic circulation and can overcome the problems associated with the free drug. Bovine serum albumin (BSA)-drug conjugates were produced with either methotrexate (MTX), a potent anticancer agent, or vancomycin (VCM), a potent antibiotic. Those conjugates were used to produce functionalized BSA nanoemulsions in a formulation composed by aqueous phase and organic phase. BSA-Folic acid (FA) conjugates were also produced allowing specific folate receptor (FR) mediated targeting of cancer cells (KB cell line). All conjugates had similar effects either in solution or in the form of nanoemulsions: BSA-MTX as anti-proliferative over Caco-2 cell line and BSA-VCM as lower minimum inhibitory concentration (MIC) comparatively to VCM solution on Staphylococcus aureus strain Newman. The production of nanoemulsions using BSA-drug conjugates for obtaining vectors loaded with stabilized drugs offers a good, flexible template for a wide range of medical applications.

\section{Introduction}

Albumin, with a molecular weight of $66.5 \mathrm{kDa}$, is the most abundant plasma protein (35-50 g/L human serum). Small size and abundance explain why so many metabolic compounds and therapeutic drugs are transported by this protein. ${ }^{1} \mathrm{BSA}$ is widely used for drug delivery because of its medical importance, abundance, low cost, ease of purification, biodegradability, nontoxicity, nonimmunogenicity, ease to be metabolized in vivo to produce innocuous degradation products and solubility in water. ${ }^{2}$

The fabrication of albumin-based nanoparticles as controlled release drug delivery systems, using specialized nanotechnology techniques like desolvation, emulsification, thermal gelation and recently nano-spray drying, nab-technology and self-assembly has been largely investigated..$^{2-8}$ Albumin nanoparticles are biodegradable, easy to prepare and reproducible in defined sizes, and carry reactive groups on their surfaces (amino, thiol and carboxylic groups) that can be used for drug conjugation and/or other surface modifications. ${ }^{9,}{ }^{10}$ Due to a high content of charged amino acids (for example lysine, arginine, aspartate and glutamate), the albumin nanoparticles allow electrostatic adsorption of positively or negatively charged molecules. ${ }^{11}$ Albumin proteins also offer the advantage of easy attachment of ligands/drugs by covalent linkage. ${ }^{12,13}$ Moreover, albumin nanoparticles represent a promising strategy for targeted drug delivery to tumor cells. Their enhanced uptake in solid tumors is mediated by binding to albumin-binding proteins, such as membrane- associated gp60 (albondin) and "secreted protein, acidic and rich in cysteine" (SPARC). ${ }^{11}$

Here, we describe the design and construction of BSA nanoemulsions tailored for specific functions, with improved and controlled drug delivery at target sites. We synthesized several BSAdrug conjugates and produced the corresponding nanoemulsions by high pressure homogenization from an initial formulation composed by an aqueous phase and a small percentage of organic phase (vegetable oil). PEGylated surfactant was introduced in the aqueous phase of this initial formulation and small PEGylated nanoemulsions were obtained. ${ }^{14}$ These PEGylated nanoemulsions with specific functions, which were obtained by introduction of BSA-FA/drug conjugates, were demonstrated to delivery drugs in vitro efficiently.

All these characteristics of the produced nanoemulsions can overcome the problems associated with therapies using free drugs, by increasing the half-life of drugs in systemic circulation, protecting them from degradation and also reducing potential side effects due to the specific cell targeting.

\section{Results and discussion}

BSA-FA conjugate and nanoemulsions for specific FR-mediated endocytosis

In order to produce nanoemulsions with the capacity of specific cell/tissue targeting, we produced BSA-FA conjugates, which were subsequently introduced in nanoemulsion production. The coupling 
of FA to BSA was performed via carbodiimide linkage ${ }^{15,16}$ between the carboxylic acid groups of each drug molecules and the 30 to 35 lysines which have primary amines that can react with the amine reactive intermediate compound (Fig. 1).<smiles>Nc1nc2ncc(CNc3ccc(C(=O)NC(CCC(=O)O)C(=O)O)cc3)nc2c(=O)[nH]1</smiles>

Folic Acid (FA), 1<smiles>CN(Cc1cnc2nc(N)nc(N)c2n1)c1ccc(C(=O)NC(CCC(=O)O)C(=O)O)cc1</smiles>

Methotreaxate (MTX), 2

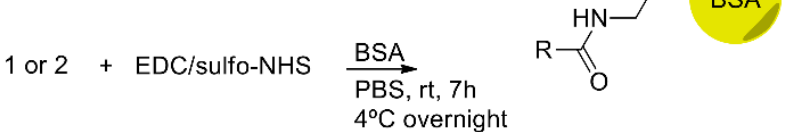

R: Folate or Methotrexate
Fig. 1 Scheme of synthesis of BSA-FA and BSA-MTX conjugates.

Our chemical synthesis conditions of conjugated BSA-FA permitted a good quantitative yield reaction that could be determined by absorption spectroscopy. The resulting conjugates contained 35 moles of FA per 1 mole of BSA, so all the available lysines residues of the BSA were coupled to a FA molecule (Supplementary Table S1).

The purified BSA-FA conjugate solution was added to BSA solution (aqueous phase) to produce FA-tagged nanoemulsions, using a high pressure homogenizer. The FA acts as targeting agent, since
FR-alpha is overexpressed on the surface of many human tumor cells. ${ }^{17}$ FA-directed nanoemulsions were produced using varying concentrations of BSA-FA conjugate and their physicochemical characteristics determined by dynamic light scattering (DLS) (Fig. 2). All samples present very similar values of particle size (around 100 $\mathrm{nm}$ ), polydispersity index (PDI) and zeta-potential consistently near zero, as previously described for the original nanoemulsions. ${ }^{14}$

The absorbance spectra of aqueous phase separated from each nanoemulsion sample was monitored $(\Lambda=350 \mathrm{~nm})$ in order to quantify FA that was not used in FA-tagged nanoemulsions production. Upon doubling the BSA-FA concentration present in the initial formulation, the quantified $\mathrm{FA}$ in aqueous phase after nanoemulsions preparation did not correlate in the same proportion. In fact, increasing BSA-FA conjugate concentration, during production, results in the successful incorporation of more FA into the nanoemulsions than expected (Supplementary Fig. S1). To determine if increasing BSA-FA conjugate for nanoemulsions preparation can more efficiently promote specific targeting, we used spectrofluorimetry to quantify nanoemulsions internalization by human mouth epidermal carcinoma cell line (KB cell line), a widely used model for evaluating FR-alpha mediated delivery. ${ }^{18}$ Fig. $3 \mathrm{~A}$ shows that FA-nanoemulsions are internalized more efficiently and to a higher extent than non-targeted nanoemulsions. However, no significant differences were observed between nanoemulsions prepared using varying quantities of BSA-FA conjugate.

Internalization assessment of nanoemulsions by confocal laser scanning microscopy, using $0.1 \mathrm{mg} / \mathrm{mL}$ of BSA-FA conjugate, confirmed that KB cells internalized more fluorescent FA-targeted nanoemulsions than cells incubated with non-targeted nanoemulsions (Fig. 3B). These results suggest that FA-nanoemulsions interact efficiently with cellular FR at the cellular surface and promote FRmediated endocytosis. This important achievement opens a wide range of possibilities for incorporating existing and validated BSA bioconjugates, that are typically short-lived in the organism, into stable nanoemulsions for targeted therapies with improved efficiency.

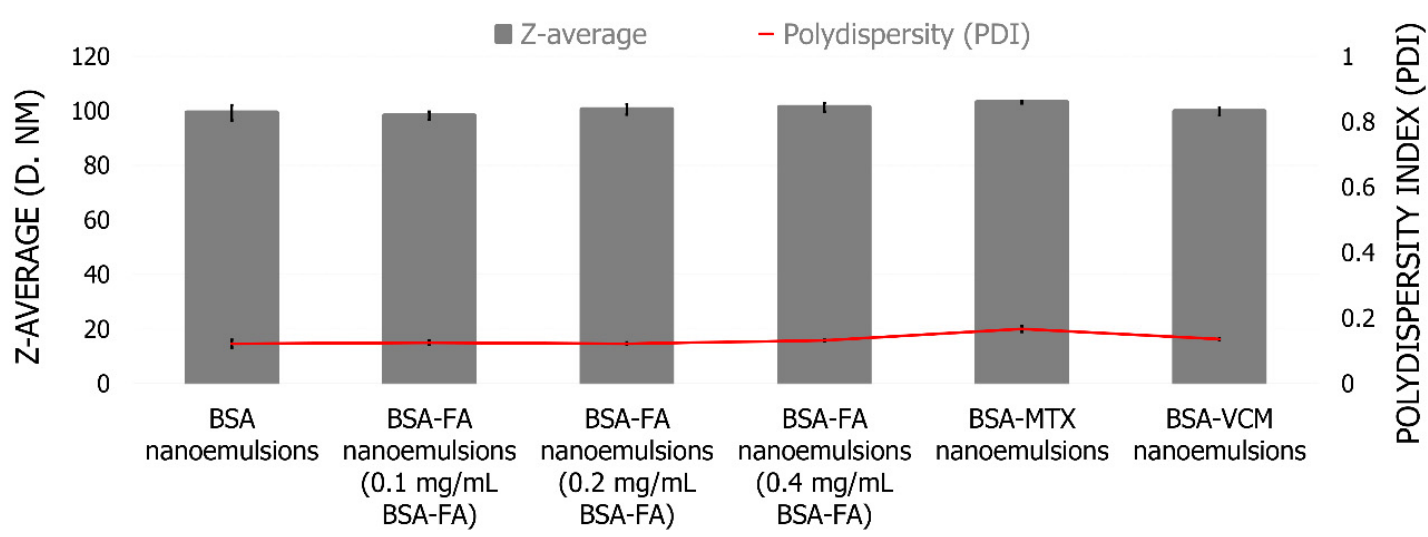

Fig. 2 Characterization in terms of size (Z-average) and PDI of BSA nanoemulsions and BSA nanoemulsions prepared using BSA-FA, BSAMTX and BSA-VCM conjugates. 


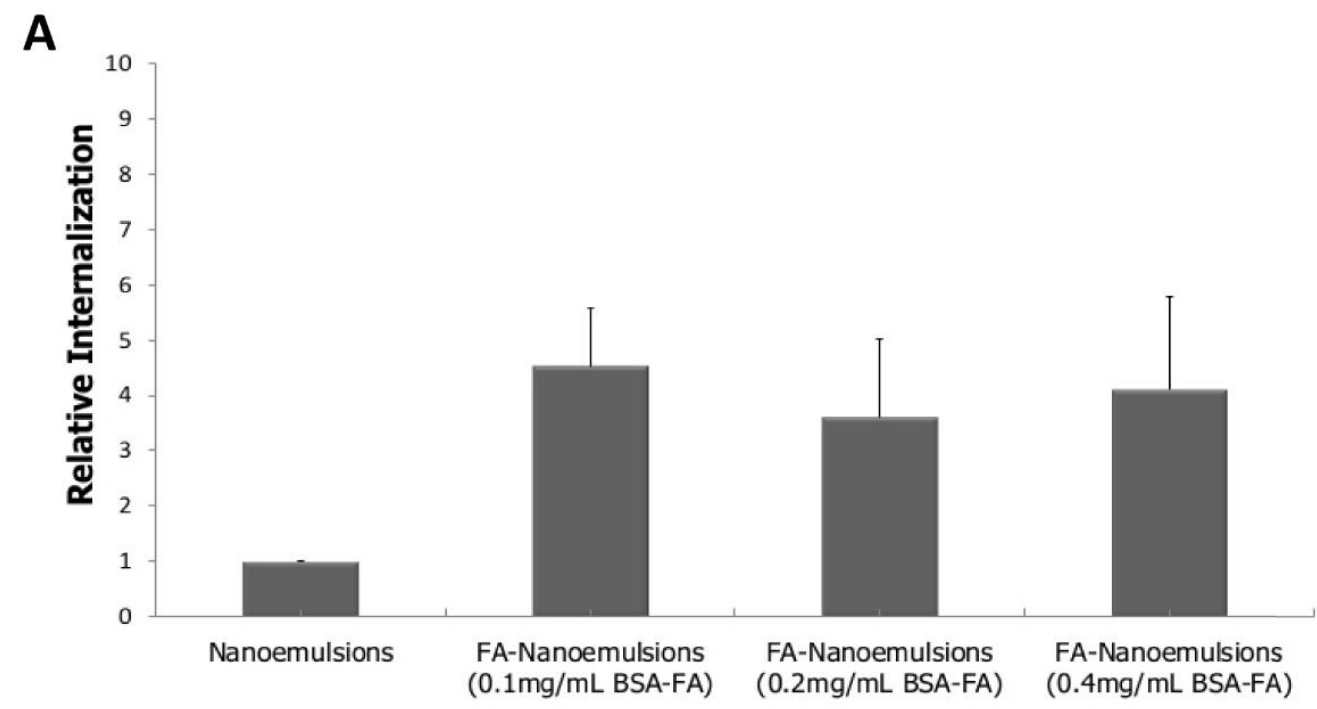

B

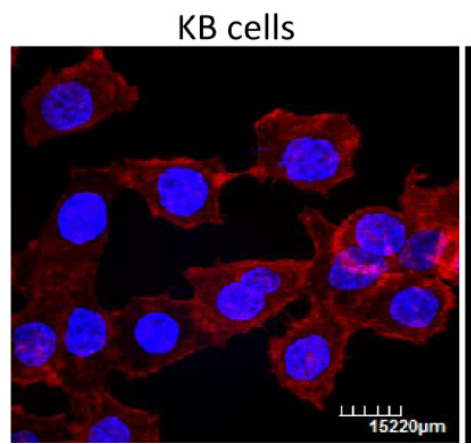
$\mathrm{KB}$ cells + Nanoemulsions

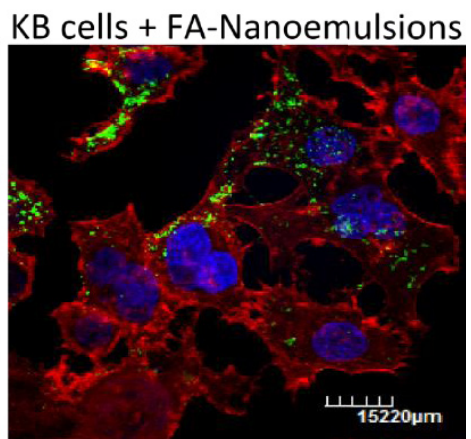

Fig. 3 Evaluation of nanoemulsions internalization using $\mathrm{KB}$ cell line. (A) Relative nanoemulsion internalization between non-targeted nanoemulsions and FA-nanoemulsions (prepared using different concentrations of BSA-FA conjugate), determined by incubation of these nanoemulsions with $\mathrm{KB}$ cells and quantification of nanoemulsions internalized using a spectrofluorimeter $(\Lambda=350 \mathrm{~nm})$. Values are the mean \pm SEM of 2 independent experiments. (B) Confocal images of fluorescent nanoemulsions internalization in KB cells. Blue: Hoescht staining of cell nucleus; Red: Alexa Fluor 568 Phalloidin staining of cytoscheleton; Green: Fluorescein isothiacianate (FITC) staining of BSA in nanoemulsions.

\section{BSA-MTX conjugate and anticancer nanoemulsions}

MTX is a potent anticancer agent of proven benefit in the treatment of acute lymphocytic leukemia, lymphomas, choriocarcinoma, some solid tumors and was also introduced for the therapy of rheumatological disorders. ${ }^{19}, 20$ However, long-term treatment and clinical studies have revealed that the curative effect of MTX on cancers was limited because it led to toxic dose-related side effects and because of the drug resistance of the tumor cells. The improvement of the pharmacokinetic behavior of MTX is therefore important to enhance its tumor targeting ability, reduce its toxicity and eventually overcome cell resistance. The development of MTX conjugates, as well as of nanoparticles containing the drug encapsulated could be a successful approach to overcome these problems. ${ }^{21-23}$

Albumin is a versatile protein carrier for drug targeting and for improving the pharmacokinetic profile of small drug molecules, peptides or protein based drugs, ${ }^{13,24}$ so we tested its conjugation with this potent anticancer drug and subsequently encapsulation into nanoemulsions.

The BSA-MTX conjugate ${ }^{25}$ was also prepared via carbodiimide linkage (Fig. 1) and the solution obtained after dialysis showed a ratio of 11 moles of MTX per 1 mole of BSA, with estimated $31 \%$ of the free lysines residues of the protein coupled with MTX (Supplementary Table S1). Purified BSA-MTX conjugate was introduced in the initial formulation at a $1: 2$ ratio $(\mathrm{m} / \mathrm{m})$ relative to the BSA protein, and nanoemulsions were produced by high pressure homogenization and further characterized (Fig. 2). These nanoemulsions also present very similar values of particle size (around $100 \mathrm{~nm}$ ) and PDI and a drug concentration encapsulated of $788 \mu \mathrm{M}$. Zeta-potential values did not suffer significant variations from the empty nanoemulsions. The development of BSA-MTX conjugate and nanoemulsions containing this conjugate can overcome the problems associated to this drug, namely their important side effects. These nanoemulsions produced present suitable characteristics as drug delivery systems for administration by 


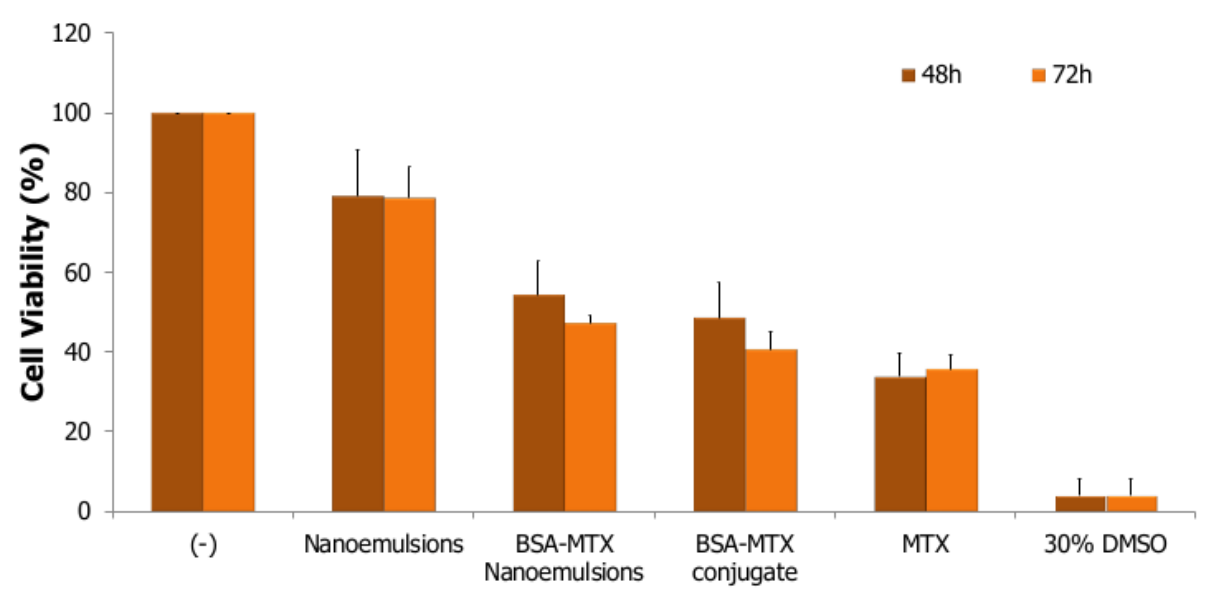

Fig. 4 Caco-2 cell line viability after $48 \mathrm{~h}$ and $72 \mathrm{~h}$ of contact with $150 \mu \mathrm{g} / \mathrm{mL}$ of nanoemulsions with and without $3.15 \mu \mathrm{M}$ of MTX, BSA-MTX conjugate at same concentration of MTX and MTX alone, compared with cells (negative control) and cells incubated with $30 \%$ of DMSO (death control), determined by MTS assay. Values are the mean \pm SEM of 2 independent experiments.

intravenous administration, e.g. small size $(\approx 100 \mathrm{~nm})$ and PEGylated surface, which can increase the half-life of drugs in systemic circulation. ${ }^{14}$ Additionally, the presence of albumin promotes targeting to the tumor site with these nanoemulsions. We then compared the cytotoxicity induced by nanoemulsions containing MTX and conjugate BSA-MTX with MTX alone at same concentration in human colonic epithelial cell line (Caco-2 cell line). Fig. 4 shows that nanoemulsions loaded with MTX, BSA-MTX conjugate and MTX alone demonstrated very similar values of cell viability, supporting another important characteristic for these nanoemulsions, the effective release of the drug from nanoemulsions in vitro.

\section{BSA-VCM conjugate and antimicrobial nanoemulsions}

Staphylococcus aureus is a major human pathogen that causes a variety of diseases with significant morbidity and mortality. ${ }^{26,} 27$ Glycopeptide antibiotics, such as VCM, are considered first-line drugs for treatment of infections caused by methicillin-resistant $S$. aureus (MRSA) and also in patients who are allergic to penicillins. ${ }^{28}$ However, the occurrence of vancomycin-resistant microbial pathogens has been increasing, posing a serious problem worldwide. ${ }^{29,} 30$

In order to protect VCM activity when this drug is administered intravenously, we also prepared BSA-VCM conjugates and thereafter produced nanoemulsions, which present suitable characteristics for this particular application.

A simple and novel BSA-VCM conjugate was synthesized via thiol-maleimide addition $^{31}$ (Fig. 5). The conjugate was obtained by reacting in the first step the VCM with the linker 4- $(N-$ maleimidomethyl) cyclohexane-1-carboxylic acid 3-sulfo- $N$ hydroxysuccinimide ester sodium salt, which is reactive between the free amino group of the VCM and the succinimide group of the linker. The second step of the reaction is carried out between the maleimideactivated VCM with the only free thiol group present in BSA in Cys34.

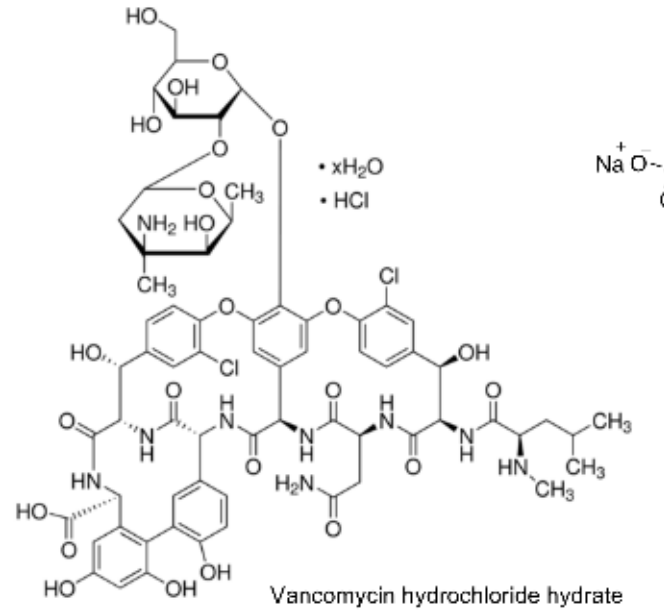

Vancomycin hydrochloride hydrate

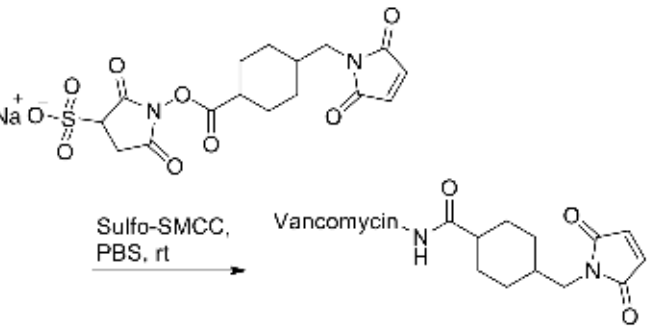

Maleimide-activated Vancomycin

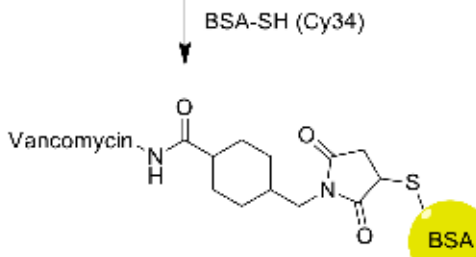

BSA-Vancomycin Conjugate via Michael addition

Fig. 5 Scheme of synthesis of BSA-VCM conjugate. 


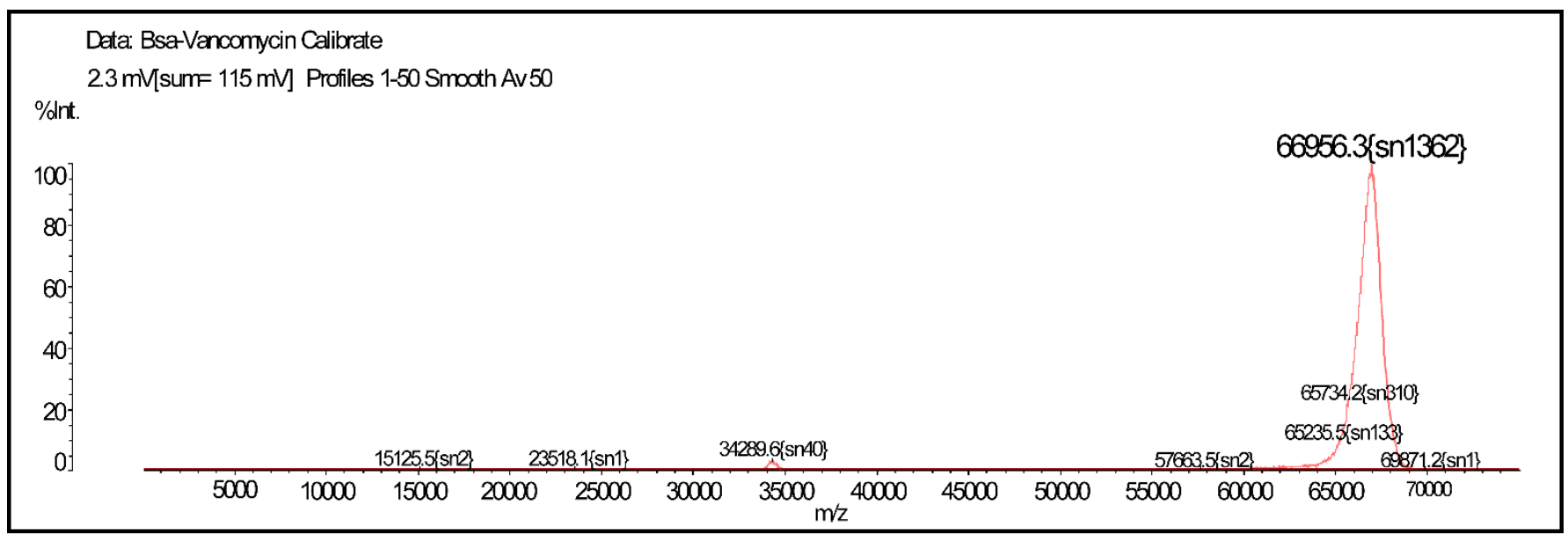

Fig. 6 MALDI spectrum of the BSA-Vancomycin conjugate. Analyses were performed on an Axima LNR system (Kratos Analytical, Shimadzu, Manchester, UK) equipped with a nitrogen laser (337 nm). The mass range from 2000 to 160000 Da was recorded by using the linear mode. Standard proteins including desalted BSA were used for external calibration of the spectra. Dihydroxybencoic acid (DHB) was used as MALDI matrix.

The resulting conjugate was analyzed by MALDI-TOF spectrometry which showed that $36 \%$ of the BSA molecules were modified with a molecule of VCM (Fig. 6). The purified conjugate solution showed a ratio of 0.28 moles of VCM per 1 mole of BSA (Supplementary Table S1) and was used for further production of functionalized BSA nanoemulsions.

BSA-VCM conjugate nanoemulsions, containing the PEGylated surfactant, were characterized in terms of size (Fig. 2) and the amount of drug encapsulated was quantified by spectroscopy ${ }^{32}$ (45 $\mu \mathrm{g} / \mathrm{mL})$. Zeta-potential values were very similar to what was previously recorded.

The determination of MIC (the lowest concentration of an antimicrobial that will inhibit the visible growth of a microorganism after overnight incubation) of VCM, BSA-VCM conjugate solution and BSA nanoemulsions loaded with VCM was performed using $S$. aureus strain Newman (Table 1).

Table 1 The MIC of BSA solution, VCM solution, BSA-VCM conjugate solution, BSA nanoemulsions and BSA-VCM nanoemulsions for $S$. aureus Newman

\begin{tabular}{cc}
\hline Samples & MIC $(\boldsymbol{\mu g} / \mathbf{m L})$ \\
\hline BSA solution & no effect \\
\hline VCM solution & 2.5 \\
\hline BSA-VCM conjugate & 1.5 \\
\hline BSA nanoemulsions & no effect \\
\hline BSA-VCM nanoemulsions & 2 \\
\hline
\end{tabular}

These results demonstrate that the BSA-VCM conjugate as well as the derived nanoemulsions potentiate the antimicrobial activity of VCM in this $S$. aureus strain, demonstrating effective release of drug from these systems. Moreover, VCM encapsulated in nanoemulsions present advantages for administration and protection of the active principle that should potentiate therapeutic efficiency in vivo.

\section{Experimental}

\section{Chemical modification of BSA with FA and MTX}

The conjugation process of drug to BSA (Sigma-Aldrich, USA) is described as follows: Briefly, each drug molecule, FA (SigmaAldrich, USA) or MTX (Sigma-Aldrich, USA) was dissolved in phosphate buffered saline (PBS, pH 7.4) and 'activated' with 5-fold excess of N-(3-dimethylaminopropyl)-N'ethylcarbodiimide hydrochloride (Sigma-Aldrich, USA) and 1fold excess of N-hydroxysulfosuccinimide (Sigma-Aldrich, USA) for $2 \mathrm{~h}$ at room temperature. To each activated drug molecule was then added $10 \mathrm{mg} / \mathrm{mL}$ of BSA and left reacting during 8-10 hours, starting all the reactions in moles ratio of 75:1 drug to BSA. Excess of drug molecules and by-products were removed from conjugated protein using dialysis against PBS (pH 7.4) for 8 days.

\section{Chemical modification of BSA with VCM}

The synthetic pathway of BSA-VCM was performed as follows: To a solution of VCM (Sigma-Aldrich, USA) (40 mg, $0.27 \mathrm{mM}$ ) in $\quad$ PBS $(\mathrm{pH}$ 7.4), the linker 4-(NMaleimidomethyl)cyclohexane-1-carboxylic acid 3-sulfo-Nhydroxysuccinimide ester sodium salt (sulfo-SMCC) (SigmaAldrich, USA) (16 mg, $0.35 \mathrm{mM}$ ) was added and the mixture was left stirring during $2 \mathrm{~h}$ at room temperature. After this time, BSA was added in a moles ratio relatively to the VCM of $1: 1$. The reaction mixture was centrifuge (5000 g, $40 \mathrm{~min}$ ) using the centricon tubes (molecular-weight cut-off of $3 \mathrm{kDa}$, Amicon Ultra-15, Millipore) and redispersed with PBS. For MALDITOF analysis the BSA-VCM conjugated and a solution of BSA were desalted using PD-10 desalting columns. 


\section{Quantitation of drug bound to BSA}

Quantitation of drug was performed by measuring the absorbance at the maximum wavelength of each drug (FA at 350 $\mathrm{nm}$ and MTX at $372 \mathrm{~nm}$ ). Quantification of VCM conjugated with BSA was performed using MALDI-TOF spectrometry.

\section{Quantitation of BSA}

Quantitation was performed by Bio-Rad protein assay (Bio-Rad Laboratories, USA) based on the shift of absorbance maximum for an acidic solution of Coomassie Brilliant Blue G-2SO at 595 $\mathrm{nm}$ when binding to protein occurred.

\section{BSA nanoemulsion preparation}

The preparation of BSA nanoemulsions was achieved by emulsion using a high-pressure homogenizer (APV-2000, Denmark). BSA at concentration of $10 \mathrm{mg} / \mathrm{mL}$ and PEGylated surfactant at concentration of $5 \mathrm{mg} / \mathrm{mL}$, previously dissolved in PBS ( $\mathrm{pH}$ 7.4), were emulsified with the organic solvent (vegetable oil) by subjecting the mixture to high pressure homogenization. BSA-FA conjugate, previously prepared, was incorporated at different ratio $(\mathrm{m} / \mathrm{m})$, relatively to $\mathrm{BSA}$, to aqueous phase and nanoemulsions tagged with FA were obtained. BSA-MTX conjugate solution, previously prepared, was also added to the aqueous phase at a $1: 2$ ratio $(\mathrm{m} / \mathrm{m})$ relative to the BSA protein. BSA-VCM conjugate solution, previously prepared, was used as total aqueous phase for nanoemulsions production. After synthesis, the nanoemulsions-containing phase was collected by centrifugation (1000 $\mathrm{g}$ and during 45 minutes) using centricon tubes (molecular weight cut-off of 100 $\mathrm{kDa}$ ) (Amicon Ultra-15, Millipore). The free protein conjugates in the aqueous phase after separation was analyzed for quantification of protein and of different ligands conjugated with BSA.

\section{Determination of size and polydispersity index}

The nanoemulsions were analyzed for their size distribution, at $\mathrm{pH} 7.4$ (PBS buffer) and at $25^{\circ} \mathrm{C}$, using dynamic light scattering in a Malvern zetasizer NS, by photon correlation spectroscopy (PCS). The protein concentration was kept constant at 10 $\mathrm{mg} / \mathrm{mL}$. The values for viscosity and refractive index were taken as $0.890 \mathrm{cP}$ and 1.330 , respectively. Each sample was measured in triplicate and results are presented as mean value \pm standard deviation.

\section{Cells and culture conditions}

Human mouth epidermal carcinoma cells (KB cell line) (ATCC, CCL-17) and human colonic epithelial cells (Caco-2 cell line) (ATCC, HTB-37) were obtained from American Type Culture Collection (LGC Standards, UK). KB cells line were maintained in Roswell Park Memorial Institute (RPMI)-1640 Medium (Sigma-Aldrich, USA) supplemented with 10\% (v/v) of fetal bovine serum (FBS) (Sigma-Aldrich, USA), 1\% (v/v) of penicillin/streptomycin solution (Sigma-Aldrich, USA), $2 \mathrm{mM}$ of L-glutamine (Sigma-Aldrich, USA) and $2 \mathrm{~g} / \mathrm{L}$ of sodium bicarbonate (Sigma-Aldrich, USA). Caco-2 cells line were routinely grown in Dulbecco's Modified Eagle's Medium
(DMEM) (Sigma-Aldrich, USA) containing $2 \mathrm{mM}$ of Lglutamine, $4.5 \mathrm{~g} / \mathrm{L}$ of glucose, $1.5 \mathrm{~g} / \mathrm{L}$ of sodium bicarbonate, supplemented with $20 \%(\mathrm{v} / \mathrm{v})$ of FBS, $1 \%(\mathrm{v} / \mathrm{v})$ of penicillin/streptomycin solution and $1 \%(\mathrm{v} / \mathrm{v})$ of Minimum Essential Medium (MEM) non-essential amino acids (SigmaAldrich, USA). All these adherent cell lines were grown in T75 flasks (TPP, Switzerland) as a monolayer.

\section{Quantification of nanoemulsions internalization}

$\mathrm{KB}$ cells were seeded in 6-well plates (TPP, Switzerland) at a density of $2 \times 10^{5}$ cells/well and incubated overnight to promote cell adhesion. Solutions of nanoemulsions with and without BSA-FA conjugate were prepared in folate-free Hanks' Balanced Salt solution (HBSS medium) (Lonza, Belgium). Cells were washed twice with PBS ( $\mathrm{pH}$ 7.4) and nanoemulsions solutions at concentration of $300 \mu \mathrm{g} / \mathrm{ml}$ were added to monolayer of cells and incubated at $37^{\circ} \mathrm{C}$ during 2 hours. After three further washing steps with cold PBS, cells were lysed with one solution of Tris- $\mathrm{HCl}$ buffer at $10 \mathrm{mM}$ and the fluorescence of these solutions was then analyzed with an Horiba Jobin Yvon Spex Fluorolog-3 spectrofluorimeter using the $\lambda \operatorname{exc}=480 \mathrm{~nm}$, scanning within a wavelength range of $490 \mathrm{~nm}$ to $650 \mathrm{~nm}$. Calibration curves of each solution of tested nanoemulsions were also measured.

\section{Observation of nanoemulsions internalization by confocal microscopy}

KB cells were seeded at a density of $5 \times 10^{4}$ cells/well and incubated overnight to promote cell adhesion. Solutions of nanoemulsions with and without BSA-FA conjugate were prepared in HBSS medium. Cells were washed twice with PBS buffer and then $300 \mu \mathrm{g} / \mathrm{mL}$ nanoemulsion suspensions were added to the monolayer, which was incubated at $37^{\circ} \mathrm{C}$ for 2 hours. After three further washing steps with cold PBS buffer, cells were fixed with $500 \mu \mathrm{L}$ of paraformaldehyde $4 \%(\mathrm{v} / \mathrm{v})$ for 30 minutes. The cells were further washed twice with PBS and incubated with Triton $0.1 \%(\mathrm{v} / \mathrm{v})$ solution, in order to promote the cells permeabilization for phalloidin staining. After two washing steps with PBS, fixed cells were incubated with 5 units/mL of Alexa Fluor 568 Phalloidin (Molecular Probes by Life Technologies, USA). The coverslips were put on slides coated with $2 \mu \mathrm{L}$ of Permafluor (Thermo Scientific, United Kingdom) with $5 \mu \mathrm{g} / \mathrm{mL}$ of Hoescht (Molecular Probes by Life Technologies, USA). Microscopic observations were done with inverted Zeiss confocal laser scanning microscope (CLSM; Olympus Fluoview FV1000).

\section{Cell viability assay}

Cell viability was studied using the Promega CellTiter 96 $\AA$ AQueous Non-Radioactive Cell Proliferation (MTS) assay (Promega, USA). Caco-2 cells were seeded in 96-well tissue culture polystyrene plates (TPP, Switzerland) at a density of $1 \times 10^{4}$ cells/well and incubated overnight to promote cell adhesion. Cells were incubated with $150 \mu \mathrm{g} / \mathrm{mL}$ of nanoemulsions with and without $3.15 \mu \mathrm{M}$ of MTX, with BSAMTX conjugate at same concentration of MTX and with MTX 
alone. Two incubation times (48 and 72 hours) were tested and then a MTS mixture $(20 \mu \mathrm{L} /$ well $)$ was added and the cells incubated for 4 hours at $37^{\circ} \mathrm{C}$. After this period, the plates were placed on a microwell plate reader Spectra MAX 340PC and the absorbance of the formazan product was read at $490 \mathrm{~nm}$. Cell viability was expressed as a percentage relative to the negative control (untreated control cells).

\section{Determination of the MIC of BSA-VCM conjugate and BSA- VCM nanoemulsions}

MIC was determined by the tube dilution test. Briefly, $2 \mathrm{~mL}$ of Luria-Bertani (LB) medium was inoculated with an overnight culture of $S$. aureus Newman to give an optical density at 600 $\mathrm{nm}$ of 0.01 . Concentrations of several VCM conjugates, nanoemulsions prepared using conjugates and respective controls, ranging from 0.25 to $3 \mu \mathrm{g} / \mathrm{ml}$, were added to the diluted suspensions in 24-well plates, which were incubated for $24 \mathrm{~h}$ at $37^{\circ} \mathrm{C}$. The concentration of VCM in the first well in the series with no sign of visible growth was reported as the minimal inhibitory concentration. The experiments were performed for two biological samples.

\section{Conclusions}

We describe the design and straightforward production by high pressure homogenization of functionalized nanoemulsions using chemically modified BSA, with a wide range of applications. Including BSA-FA conjugate in the initial formulation allowed the production of FA-tagged nanoemulsions that demonstrated specific FR-mediated targeting in KB cancer cell line. BSA conjugated with two different drugs (MTX and VCM) also demonstrated good results when applied in vitro. MTX encapsulated in nanoemulsions present numerous advantages to using the drug alone or the MTXbioconjugates, such as protection of the bioactive compound from degradation in vivo and improved half-life in systemic circulation. The combination of such advantages would overcome severe side effects associated with MTX treatment. Nanoemulsions prepared with BSA-VCM led to a significant reduction of MIC in S. aureus strain Newman. These results showed that the BSA-VCM bioconjugate, alone or formulated in a nanoemulsion, can potentiate the antimicrobial effect of this drug, reducing the need for high dose therapy and associated problems.

Thus, the development of these BSA-FA/drugs conjugates and their use in the production of BSA nanoemulsions previously developed, which are small and stealth nanoparticles, ${ }^{14}$ allowed the construction of functionalized, effective nanoemulsions. The approach here described results in functionalized nanoemulsions that are suitable for drug delivery application in a very wide range of clinical settings.

\section{Acknowledgements}

Ana Loureiro (SFRH/BD/81479/2011) holds a scholarship from Fundação para a Ciência e a Tecnologia (FCT). Gonçalo J. L. Bernardes is a Royal Society University Research Fellow at the
Department of Chemistry, University of Cambridge and an Investigador FCT at the Instituto de Medicina Molecular, Faculdade de Medicina da Universidade de Lisboa. This work has received funding from the European Union Seventh Framework Programme (FP7/2007-2013) under grant agreement NMP4-LA-2009-228827 NANOFOL. This work was supported by FEDER through POFC - COMPETE and by Portuguese funds from FCT through the project PEstOE/BIA/UI4050/2014.

\section{Notes and references}

${ }^{a}$ CEB - Centre of Biological Engineering, Department of Biological Engineering, University of Minho, Campus de Gualtar, 4710-057 Braga, Portugal

${ }^{b}$ CBMA - Centre of Molecular and Environmental Biology, Department of Biology, University of Minho, Campus of Gualtar, 4710-057 Braga, Portugal

${ }^{c}$ ITQB - Instituto de Tecnologia Química e Biológica, Universidade Nova de Lisboa, Avenida da República, Estação Agronómica Nacional, 2780157 Oeiras, Portugal

${ }^{d}$ Department of Chemistry, University of Cambridge, Lensfield Road, Cambridge, CB2 1EW, United Kingdom

${ }^{e}$ Instituto de Medicina Molecular, Faculdade de Medicina da Universidade de Lisboa, Av. Prof. Egas Moniz, 1649-028 Lisboa, PortugalAddress here.

* Corresponding author email: artur@deb.uminho.pt

$\dagger$ Present address: Institute of Polymers and Composites (IPC) and Institute of Nanostructures, Nanomodelling and Nanofabrication (I3N), University of Minho, Campus de Azurém, 4800-058 Guimarães, Portugal

Electronic Supplementary Information (ESI) available: One Supplementary Figure and one Supplementary Table. See DOI: $10.1039 / \mathrm{b} 000000 \mathrm{x} /$

1. F. Kratz and B. Elsadek, J. Control. Release, 2012, 161, 429-445.

2. A. O. Elzoghby, W. M. Samy and N. A. Elgindy, J. Control. Release, 2012, 157, 168-182.

3. U. Shimanovich, G. J. L. Bernardes, T. P. J. Knowles and A. CavacoPaulo, Chem. Soc. Rev., 2014, 43, 1361-1371.

4. N. Suthiwangcharoen, T. Li, L. Wu, H. B. Reno, P. Thompson and Q. Wang, Biomacromolecules, 2014, 15, 948-956.

5. T. Li, L. Wu, N. Suthiwangcharoen, M. A. Bruckman, D. Cash, J. S. Hudson, S. Ghoshroy and Q. Wang, Chem. Commun., 2009, 2869-2871.

6. N. Suthiwangcharoen, T. Li, K. Li, P. Thompson, S. You and Q. Wang, Nano Res., 2011, 4, 483-493.

7. N. Anton, J.-P. Benoit and P. Saulnier, J. Control. Release, 2008, 128, 185-199.

8. G. V. Patil, Drug Dev. Res., 2003, 58, 219-247.

9. F. Kratz, Expert Opin. Ther. Patents, 2002, 12, 433-439.

10. N. Stephanopoulos and M. B. Francis, Nat. Chem. Biol., 2011, 7, 876884.

11. A. O. Elzoghby, W. M. Samy and N. A. Elgindy, J. Control. Release, 2012, 161, 38-49. 
12. P. Zdziarski, Acta Pol. Pharm., 2000, 57, 411-413.

13. F. Kratz, J. Control. Release, 2008, 132, 171-183.

14. E. Nogueira, A. Loureiro, P. Nogueira, J. Freitas, C. R. Almeida, J. Harmark, H. Hebert, A. Moreira, A. M. Carmo, A. Preto, A. C. Gomes and A. Cavaco-Paulo, Faraday Discuss., 2013, 166, 417-429.

15. D. Bartczak and A. G. Kanaras, Langmuir, 2011, 27, 10119-10123.

16. G. T. Hermanson, 2008.

17. C. Du, D. Deng, L. Shan, S. Wan, J. Cao, J. Tian, S. Achilefu and Y. Gu, Biomaterials, 2013, 34, 3087-3097.

18. J. A. Reddy, E. Westrick, H. K. R. Santhapuram, S. J. Howard, M. L. Miller, M. Vetzel, I. Vlahov, R. V. J. Chari, V. S. Goldmacher and C. P. Leamon, Cancer Res., 2007, 67, 6376-6382.

19. B. N. Cronstein, Pharmacol Rev, 2005, 57, 163-172.

20. E. S. L. Chan and B. N. Cronstein, Arthritis Res., 2002, 4, 266-273.

21. R. Sett, H. S. Sarkar and P. K. Das, J. Antimicrob. Chemother., 1993, 31, 151-159.

22. Z. Q. Samra, S. Ahmad, M. Javeid, N. Dar, M. S. Aslam, I. Gull and M. M. Ahmad, Prep. Biochem. Biotechnol., 2013, 43, 781-797.

23. Y.-H. Chen, C.-Y. Tsai, P.-Y. Huang, M.-Y. Chang, P.-C. Cheng, C.H. Chou, D.-H. Chen, C.-R. Wang, A.-L. Shiau and C.-L. Wu, Mol. Pharmaceutics, 2007, 4, 713-722.

24. B. Elsadek and F. Kratz, J. Control. Release, 2012, 157, 4-28.

25. N. Endo, Y. Kato, Y. Takeda, M. Saito, N. Umemoto, K. Kishida and T. Hara, Cancer Res., 1987, 47, 1076-1080.

26. E. Galbusera, A. Renzoni, D. O. Andrey, A. Monod, C. Barras, P. Tortora, A. Polissi and W. L. Kelley, Antimicrob. Agents Chemother., 2011, 55, 1008-1020.

27. T. Baba, T. Bae, O. Schneewind, F. Takeuchi and K. Hiramatsu, $J$. Bacteriol., 2008, 190, 300-310.

28. M. Ge, Z. Chen, H. Russell, Onishi, J. Kohler, L. L. Silver, R. Kerns, S. Fukuzawa, C. Thompson and D. Kahne, Science, 1999, 284, 507-511.

29. Y. Cetinkaya, P. Falk and C. G. Mayhall, Clin. Microbiol. Rev., 2000, 13, 686-707.

30. P. Courvalin, Clin. Infect. Dis., 2006, 42, S25-S34.

31. B. Wängler, A. P. Kostikov, S. Niedermoser, J. Chin, K. Orchowski, E. Schirrmacher, L. Iovkova-Berends, K. Jurkschat, C. Wängler and R. Schirrmacher, Nat. Protoc., 2012, 7, 1964-1969.

32. S. M. El-Ashry, F. Belal, M. M. El-Kerdawy and D. R. El Wasseef, Microchim. Acta, 2000, 135, 191-196. 